\title{
Embedded Palmprint Recognition System on Mobile Devices
}

\author{
Yufei Han, Tieniu Tan, Zhenan Sun, and Ying Hao \\ Center for Biometrics and Security Research \\ National Labrotory of Pattern Recognition,Institue of Automation \\ Chinese Acdamey of Sciences \\ P.O. Box 2728, Beijing, P.R. China, 100080 \\ \{yfhan, tnt, znsun, yhao\}@nlpr.ia.ac.cn
}

\begin{abstract}
There are increasing requirements for mobile personal identification, e.g. to protect identity theft in wireless applications. Based on built-in cameras of mobile devices, palmprint images may be captured and analyzed for individual authentication. However, current available palmprint recognition methods are not suitable for real-time implementations due to the limited computational resources of handheld devices, such as PDA or mobile phones. To solve this problem, in this paper, we propose a sum-difference ordinal filter to extract discriminative features of palmprint using only $+/$ - operations on image intensities. It takes less than $200 \mathrm{~ms}$ for our algorithm to verify the identity of a palmprint image on a HP iPAQ PDA, about 1/10 of state-of-the-art methods ' complexity, while this approach also achieves high accuracy on the PolyU palmprint database. Thanks to the efficient palmprint feature encoding scheme, we develop a real-time embedded palmprint recognition system, working on the HP PDA.
\end{abstract}

\section{Introduction}

There are about 1.5 billion mobile devices currently in use. With fast development of wireless network and embedded hardware, people enjoy the convenience of mobile commerce, mobile banking, mobile office, mobile entertainment, etc. However, at the same time, the risk of identity theft is increasing. The mostly used identity authentication method is password, but it can be cracked or forgotten. So biometrics is emerging to enhance the security of digital life. Fingerprint, face and voice recognition have been put into use on embedded mobile devices such as PDA and mobile phones. But they still have many disadvantages. For fingerprint recognition, only a small portion of mobile devices have a fingerprint sensor, and some people do not have clear fingerprints [1]. Face and voice recognition are sometimes not accurate and robust enough for personal recognition [1]. In contrast, palmprint could supply an alternative way for mobile authentication when these methods fail. According to work $[2,3]$, large areas of human palms supply enough and robust representative features for identity authentication. Furthermore, palmprint recognition is testified to achieve high accuracy in real world use based on low-resolution ( $<100$ dpi) images. As a result, using built-in cameras of PDA or mobile phones, it is possible to capture palmprint images adequate for further recognition. 
Various palmprint recognition approaches have been proposed in the literatures [112]. Almost all available algorithms involve thousands of large-size convolutions or filtering, i.e. millions of multiplication operations of floating points. Although these algorithms can work well on PC, most embedded mobile devices can not afford such complex computations because of the limited computational resources on handheld apparatus. To the best of our knowledge, there is no work reported on palmprint recognition for embedded systems. We think the main bottleneck is the limited computational resources of mobile devices. To attack this problem, we propose a novel fast palmprint feature encoding algorithm, only involving $+/$ - integer operations of pixel intensities, to make palmprint recognition applicable for mobile devices. The basic idea of our method is based on qualitative comparison of the average intensities of two elongated and orthogonal palmprint image regions, namely ordinal features [2]. Simple and fast as it is, our algorithm obtains high accuracy on PolyU palmprint database [13]. Based on the proposed approach, we develop a real-time palmprint recognition system, working on a HP rx3715 PDA.

The rest of the paper is organized as follows. We represent the total framework of the proposed system in Section 2. In Section 3, our fast palmprint encoding scheme is described. In Section 4, experiments are designed to test performance of the system. Section 5 concludes the paper.

\section{System Overview}

The architecture of the proposed system is denoted in the following diagram (see Fig. 1). We adopt a HP iPAQ rx3715 PDA as a computing platform of the system. This device contains a low-power 400MHZ StrongARM processor which lacks floating point unit. Besides, it equips with a built-in digital camera with the resolution of $100 \mathrm{dpi}$, which is used to capture palmprint images. The whole system is implemented using Microsoft Embedded Visual $\mathrm{C}++3.0$ under Windows CE operating system. Two most important components of the system are image capture and feature encoding module. The former aims to obtain normalized palmprint images, decreasing the effect of image translation, rotation and scale variations. The latter derives a compact description of each palmprint image for further feature matching in a high speed.

After a user starts the system, the built-in camera is activated automatically. A continually updated preview frame (see Fig. 2 (a)) on screen can be used to adjust the distance and position between the user's palm and the camera. During image capturing, the root region of the middle finger, forefinger and ring finger is required to be aligned with the top border of the frame. The left and right borders of the frame are required to be aligned with the corresponding borders of the central region of a palm. Then images of the central region are obtained with little rotation, translation and scale variation. In the captured image, a fixed size sub-image is cropped from it at a fixed position, which is used for feature extraction, namely region of interest (ROI), as shown in Fig. 2(b). A mobile user could control his right or left hand to capture the palmprint image of left or right hand. 


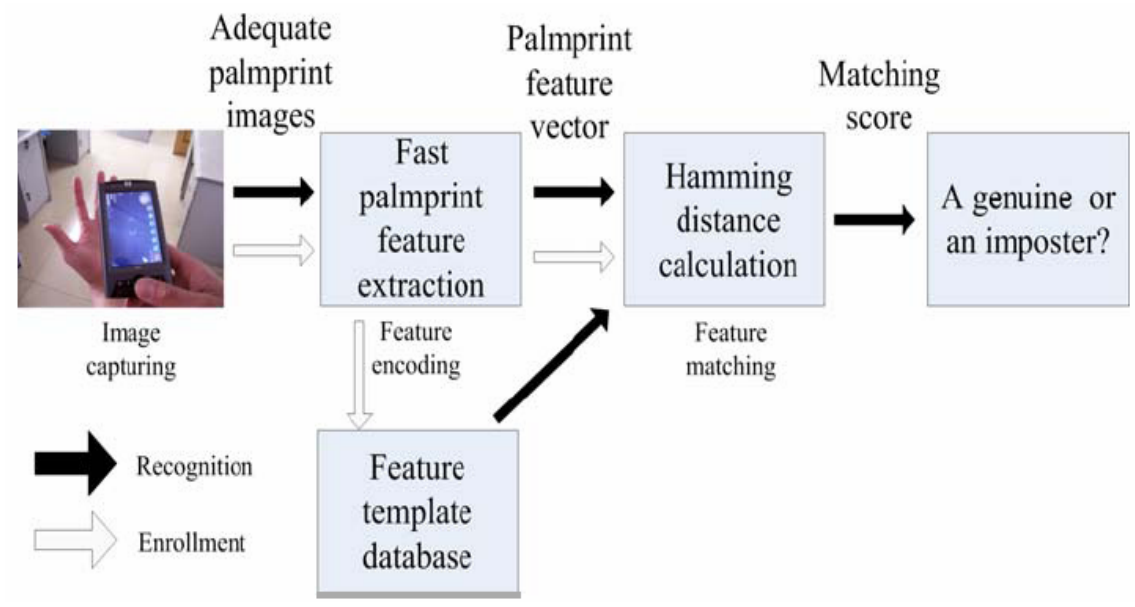

Fig. 1. System framework

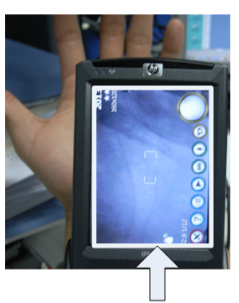

Image preview frame

(a)

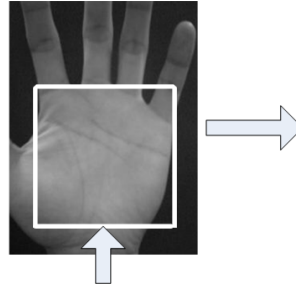

Image preview frame

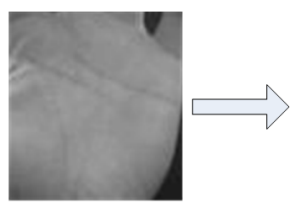

Captured palmprint image

(b)

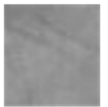

Region of interests

Fig. 2. An example of ROI generation. (a) Image preview frame;(b) Generation of ROI.

During enrollment, one user could choose either of his palms to be registered in the system. For each registered palm, the system captures three palmprint images. In our application, the size of each captured image is $160 \times 120$, and a $80 \times 80$ subimage is extracted as ROI. After feature extraction, three feature templates are compared with each other using hamming distance. Only if all obtained hamming distances are lower than a given threshold, the feature templates can be stored into a $256 \mathrm{MB}$ SD card of PDA, which is used as the feature template database. Otherwise, the system will prompt that the enrollment procedure fails. During recognition, each input palmprint feature is compared with all feature templates stored in the system by fast hamming distance matching. If the minimal hamming distance is higher than a threshold, the user is classified as an imposter; otherwise, he is accepted as a registered user. 


\section{Sum-Difference Ordinal Filter}

In our previous work, we proposed the orthogonal line ordinal feature (OLOF) [2] to represent negative line features of palmprints efficiently. The orthogonal line ordinal filter is designed as follows [2]:

$$
F(x, y, \theta)=G(x, y, \theta)-G(x, y, \theta+\pi / 2)
$$

where

$$
G(x, y, \theta)=\exp \left[-\left(\frac{x \cos \theta+y \sin \theta}{\delta_{\mathrm{x}}}\right)^{2}-\left(\frac{-x \sin \theta+y \cos \theta}{\delta_{y}}\right)^{2}\right]
$$

which is a $2 \mathrm{D}$ Gaussian filter, and $\theta$ is the orientation of the Gaussian filter. To make its shape like a line, we controlled the parameters $\delta_{x} / \delta_{y}>3$. After convolution between a local region and the ordinal filter, the filtering result is then encoded into 1 or 0 according to whether it is positive or negative. Thousands of ordinal codes are concatenated into a feature template. The OLOF describes orientation information of negative line segments in palmprints. Our fast palmprint feature encoding scheme uses simplified OLOF because the coefficients of the Gaussian function are floating point numbers and result in relative high computational cost on the PDA. So a novel ordinal filter is designed, namely, sum-difference ordinal filter (SDOF), which contains only integer coefficients instead. According to equation (1) and (2), the effective region for filtering of the OLOF [2] could be detected by projecting $F(x, y, \theta)$ to the image plane as follows:

$$
g(x, y)=\{I(x, y):|F(x, y, \theta)| \geq k, k>0\},
$$

$k$ is a fixed threshold, which is set empirically as following Eq.5.

According to Eq.3, we can obtain four discrete symmetric regions. One example is illustrated in Figure 3 (a). The square region in the example has a size of $35 \times 35$. Two regions are along the horizontal axis, while the other two along the vertical axis. Pairs along the same axis can be seen as the head and the tail of a line segment. In the SDOF, coefficients in horizontal parts are +1 , while these in vertical parts are -1 , represented as follows:

$$
\operatorname{SDOF}(x, y, \theta)= \begin{cases}1 & F(x, y, \theta) \geq k \\ -1 & F(x, y, \theta) \leq-k \\ 0 & \text { otherwise }\end{cases}
$$

where $k>0$ and $\theta$ is the orientation of the filter. According to our experiments, we choose $k$ as follows:

$$
k=0.005 \times E
$$

where $E$ is the maximum intensity of $G(x, y, \theta)$. For each ROI obtained, three sumdifference ordinal filters are performed on it as follows: 


$$
R(\theta)=\operatorname{SDOF}(x, y, \theta) * I(x, y)
$$

where $\theta=0, \pi / 6, \pi / 3 . I(x, y)$ denotes the local patch of ROI and * denotes the operator of convolution. Three bit binary codes are generated according to signs of $R(\theta)$. After the whole palmprint image filtered, numbers of these binary codes constitute a feature template, namely sum-difference ordinal code, as shown in Fig 3(b). In contrast to the orthogonal line ordinal filter [2], the feature extraction time can be reduced obviously, because there are only addition and subtraction involved in the convolution. Similarity between two feature templates is then measured by hamming distance. Simple as it is, like the orthogonal line ordinal code [2], sumdifference ordinal code also represents ordinal measures between two geometrical orthogonal line-like image regions, which is testified to be a robust and discriminating feature to describe negative line patterns in palmprint images, such as principle lines and wrinkles [2].

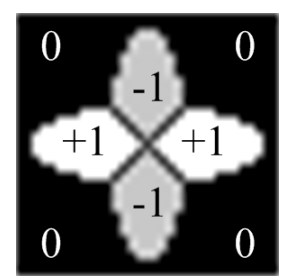

(a)

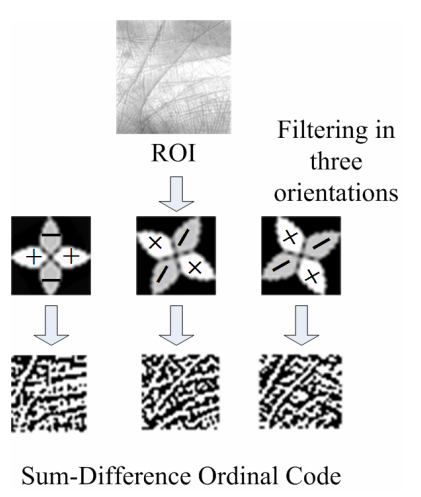

(b)

Fig. 3. An example of ordinal palmprint features extraction (a) Four symmetric regions; (b) Generation of sum-difference ordinal code

\section{Experimental Results}

This section consists of two parts. In section 4.1, we collect a palmprint image dataset using the proposed system, following the way shown in Fig. 2. Based on those images, we test performance of the system in the section, including accuracy of recognition and usability of the system. We also implement three state-of-the-art algorithms, fusion code [10], competitive code [11] and ordinal code [2] on PDA for further comparisons with the proposed sum-difference ordinal code. Besides, in section 4.2, we design a verification experiment on PolyU Palmprint Database [13] to test validity of the proposed approach in a further step.

\subsection{Performance Evaluation of the System}

We collect 400 gray-scale images from 40 subjects, with five images for each palm, Six examples are shown in Fig. 4(a). Fig. 4(b) shows an example of cropped ROI. The first three images of each palm are captured by the system as the enrolling images, 
while the other two are added further. Notably, unlike biometric systems used for entrance control, an identity authentication system of a PDA or mobile phone is usually utilized to confirm whether the current user is the owner of the mobile device. So there are only the owner's biometric features stored in the system. Therefore, we evaluate recognition accuracy of the system in terms of verification rate [14] of sumdifference ordinal code and total success rate [12], both obtained through one-to-one image matching. There are totally 800 intra-class comparisons and 3,160 inter-class comparisons. Due to large number of matching operations, all comparisons are performed on PC. We compare the average feature extraction time of the proposed algorithm with that of the other three methods [2][10][11] on the PDA. Table.1 lists performances of all four algorithms. Fig. 5 shows corresponding ROC curves. Fig.6 shows total success rate of our system varying with different threshold values. From experimental results illustrated in Tab.1 and Fig. 5, we can see that computational cost of sum-difference ordinal code is only a fraction of the others. In addition, it is the most accurate for identity verification.
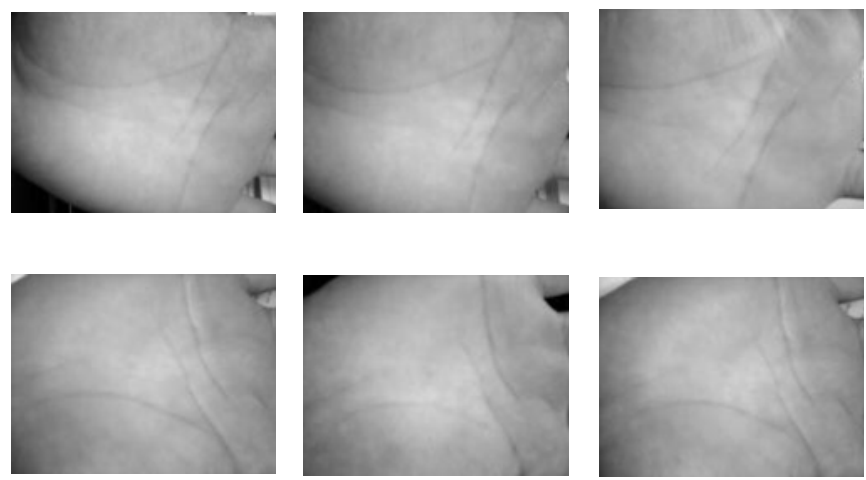

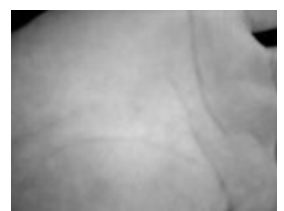

(a)
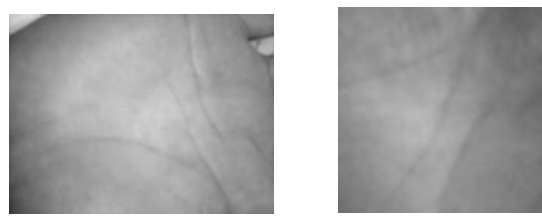

(b)

Fig. 4. Examples of palmprint images captured by the proposed system (a) Palmprint images collected by the system; (b) Region of interests

Table 1. Performance comparison on images collected by the system

\begin{tabular}{|c|c|c|c|}
\hline Algorithm & EER[14] & $d^{\prime}[14]$ & $\begin{array}{l}\text { Feature } \\
\text { extraction time } \\
\text { on PDA }\end{array}$ \\
\hline Fusion code[10] & $3.8 \%$ & 3.86 & $2296 \mathrm{~ms}$ \\
\hline Competitive code[11] & $1.1 \%$ & 4.70 & $1627 \mathrm{~ms}$ \\
\hline Ordinal code[2] & $1.02 \%$ & 4.70 & $803 \mathrm{~ms}$ \\
\hline $\begin{array}{c}\text { Sum-difference } \\
\text { ordinal code }\end{array}$ & $0.92 \%$ & 4.76 & $180 \mathrm{~ms}$ \\
\hline
\end{tabular}




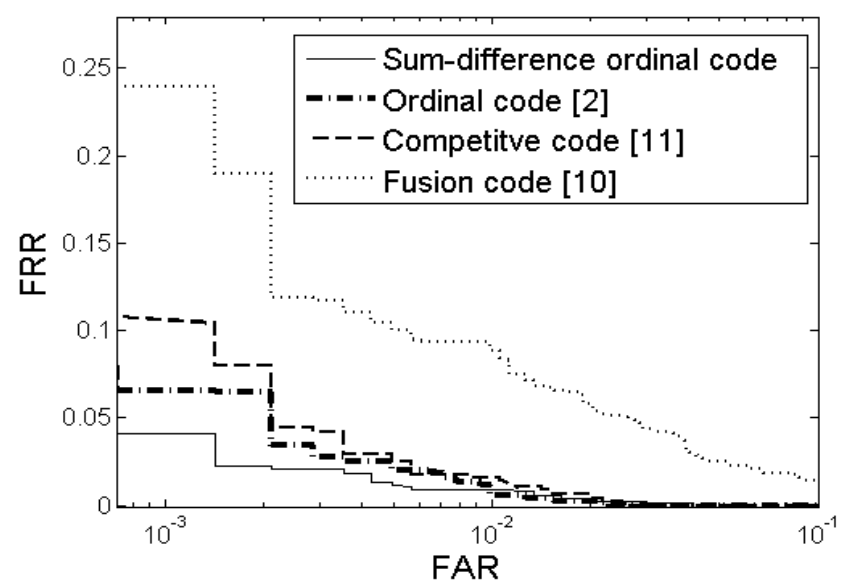

Fig. 5. ROC curves on images collected by the system

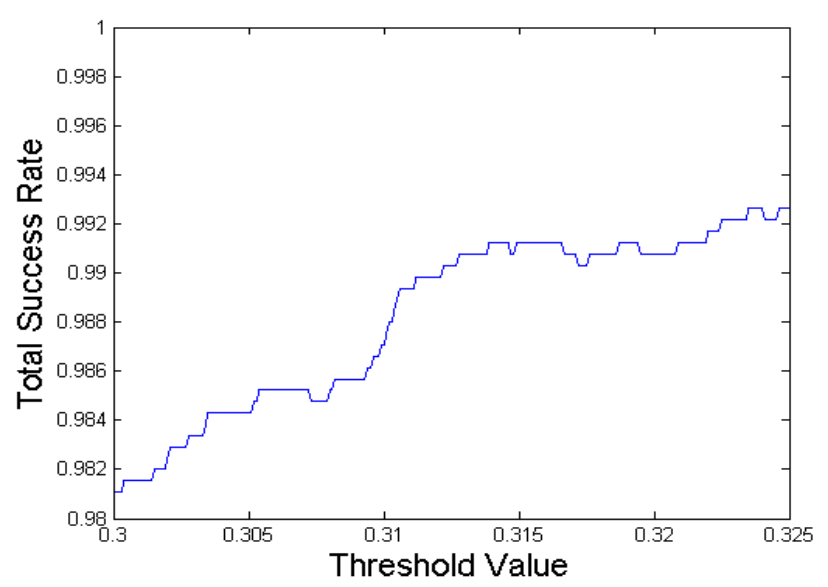

Fig. 6. Total success rate of our system

Total success rate (TSR) [12] is employed to evaluate recognition accuracy in a further step. It is calculated considering both correct accepted and correct rejected items:

$$
T S R=1-\frac{F A R \times \text { Amount of imposter }+F R R \times \text { Amount of genuine }}{\text { Total amount of matching }}
$$

As shown in Fig. 6, with proper choice of threshold value, our system achieves a total success rate of around 99\%, using the efficient feature extraction method. Due to limit to accuracy of the alignment method used in the system, described in section 2, position and scale variations still exit in captured images, which may deteriorate performance of the system. Nevertheless, based on the fast feature encoding scheme, our system can deliver a real-time recognition in relatively high accuracy on the PDA. 
Except for average feature time, we also record the enrollment duration of each subject during collecting images by the system, as shown in Fig. 7. It includes time for a complete successful enrolling procedure and depends mostly on interaction between users and the recognition system. Fast enrollment is very important for pointof-sale use. The minimum and mean enrollment duration values are 65.2 seconds and 76 seconds respectively. As we see in Fig. 7, most of subjects finish enrollment successfully in less than 78 seconds. Only one costs more than 100 seconds, due to his unfamiliarity with usage of PDA.

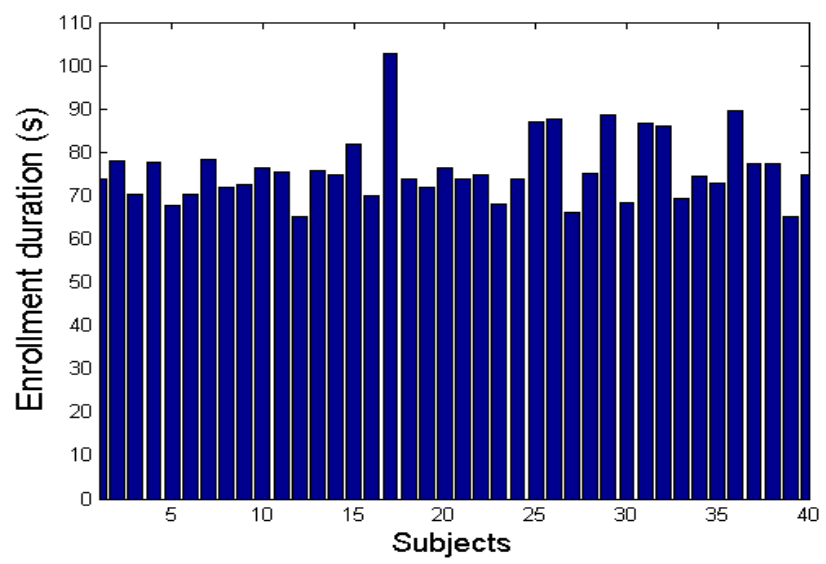

Fig. 7. Enrollment duration of 40 subjects

\subsection{Verification Experiment on the PolyU Palmprint Database}

There are totally 7,752 palmprint images from 386 different palms in the PolyU Palmprint database $2^{\text {nd }}$ version [13]. Each palm has two sessions. The average time interval between each two session is about two months. We normalize original images into $128 \times 128$ ROI images [3]. Totally 74,068 intra-class comparisons and 7,430,500 inter-class comparisons are involved. All matching procedures are performed on a common PC. Fig. 8 and Table 2 show experimental results. For each of four algorithms, we measure its average feature extraction time on the PDA, as listed in Table 2. Compared with state-of-the-art palmprint recognition algorithms [2][10][11], sum-difference ordinal code achieves significantly high accuracy. Furthermore, it costs only about 1 second for feature extraction on one ROI image, much faster than the other three opponents. ROI images in the section are larger than those in section 4.1. As a result, they contains more discriminative features. Furthermore, there is less alignment error in images of the PolyU database [12] after preprocessing [3]. Thus, all four algorithms perform better. Results demonstrate that sum-difference ordinal code is not only suitable for fast palmprint recognition on PDA, but also on PC with image capture devices introduced in [3]. 
Table 2. Performance comparison on PolyU palmpirnt image database

\begin{tabular}{|c|c|c|c|}
\hline Algorithm & EER[14] & $d^{\prime}[14]$ & $\begin{array}{l}\text { Feature } \\
\text { extraction } \\
\text { time on PDA }\end{array}$ \\
\hline Fusion code[10] & $0.21 \%$ & 5.40 & $13455 \mathrm{~ms}$ \\
\hline Competitive code[11] & $0.06 \%$ & 5.64 & $8325 \mathrm{~ms}$ \\
\hline Ordinal code[2] & $0.07 \%$ & 6.63 & $4421 \mathrm{~ms}$ \\
\hline $\begin{array}{c}\text { Sum-difference } \\
\text { ordinal code }\end{array}$ & $0.07 \%$ & 6.58 & $1127 \mathrm{~ms}$ \\
\hline
\end{tabular}

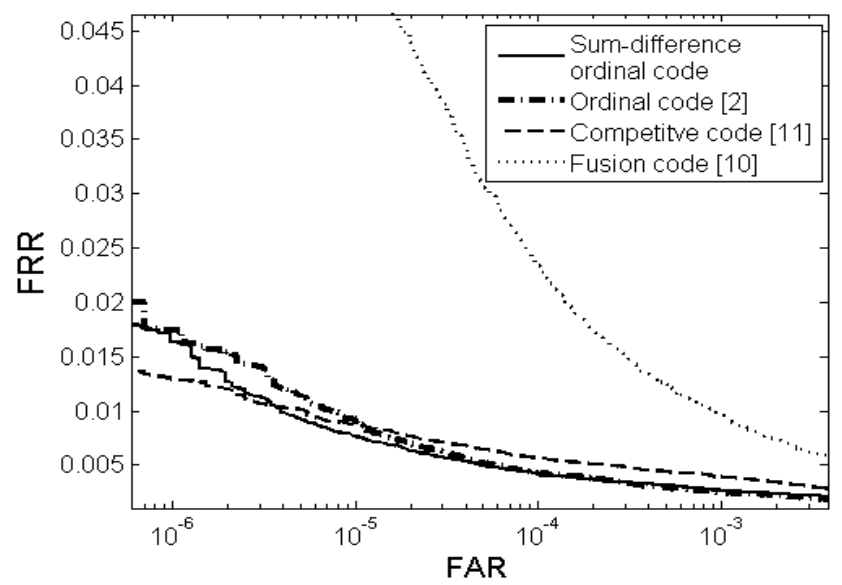

Fig. 8. ROC curves on PolyU database

\section{Conclusions and Future Work}

In this paper, we have proposed and developed a real-time palmprint recognition system for mobile devices, such as PDA. As far as we know, this is the first attempt to apply palmprint based identity check on such mobile devices. The core of the system is a fast palmprint feature encoding scheme using a sum-difference ordinal filter. The coefficients of the filter only involve $1 \mathrm{~s}$ or 0 s, so palmprint feature extraction could be implemented with only $+/$ - operations of integers. The average feature extraction time is only $180 \mathrm{~ms}$ on the HP PDA, almost ten times faster than the other three state-ofthe-art methods in the experiments. Although our method is simple, the filter is able to extract the discriminative palmprint feature --- ordinal measures [2] between a negative line pattern and its geometric orthogonal region. So it is not surprised that our method has a good performance in the verification test. Benefit from the efficient approach and human-computer interface, the proposed system achieves accurate recognition and convenient use at the same time. We can further use such a technology to protect identity of mobile applications or enhance the security of wireless communication. However, how to design a suitable image alignment method to improve accuracy of the system is not well addressed in this paper. It is an important issue in our future work. 
Acknowledgments. Experiments in the paper use the PolyU Palmprint Database ver.2 collected by the Biometric Research Center at the Hong Kong Polytechnic University. This work is funded by research grants from the National Basic Research Program (Grant No.2004CB318110), the Natural Science Foundation of China (Grant No. 60335010, 60121302, 60275003, 60332010, 69825105 60605008) and the Chinese Academy of Sciences.

\section{References}

1. Li, W., Zhang, D., Xu, Z.: Palmprint Identification by Fourier Transform. International Journal of PRAI 16(4), 417-432 (2002)

2. Sun, Z.N., Tan, T.N., Wang, Y.H., Li, S.Z.: Ordinal Palmprint Representation for Personal Identification. In: Proc. of CVPR 2005, vol. 1, pp. 279-284 (2005)

3. Zhang, D., Kong, W.K., You, J., Wong, M.: Online Palmprint Identification. IEEE Trans on PAMI 25(9), 1041-1050 (2003)

4. Zhang, D., Shu, W.: Two Novel Characteristics in Palmprint Verification: Datum Point Invariance and Line Feature Matching. Pattern Recognition 32(4), 691-702 (1999)

5. Duta, N., Jain, A.K., Mardia, K.V.: Matching of Palmprint. Pattern Recognition Letters 23(4), 477-485 (2001)

6. Lu, G., Zhang, D., Wang, K.: Palmprint Recognition Using Eigenpalms Features. Pattern Recognition Letters 24(9-10), 1463-1467 (2003)

7. Han, C.C., Cheng, H.L., Fan, K.C., Lin, C.L.: Personal Authentication Using Palmprint Features. Pattern Recognition 36(2), 371-381 (2003)

8. You, J., Kong, W.K., Zhang, D., Cheung, K.: On Hierarchical Palmprint Coding with Multi- features for Personal Identification in Large Databases. IEEE Trans on Circuit Systems for Video Technology 14(2), 234-243 (2004)

9. Zhang, L., Zhang, D.: Characterization of Palmprints by Wavelet Signatures via Directional Context Modeling. IEEE Trans on SMC-B 34(3), 1335-1347 (2004)

10. Kong, W.K., Zhang, D.: Feature-Level Fusion for Effective Palmprint Authentication. In: Zhang, D., Jain, A.K. (eds.) ICBA 2004. LNCS, vol. 3072, pp. 520-523. Springer, Heidelberg (2004)

11. Kong, W.K., Zhang, D.: Competitive Coding Scheme for Palmprint Verification. In: Proc. of the 17th ICPR, vol. 1, pp. 520-523 (2004)

12. Connie, T., Jin, A.T.B., Ong, M.G.K., Ling, D.N.C.: Automated palmprint recognition system. Image and Vision Computing 23, 501-515 (2005)

13. PolyU Palmprint Database, http://www.comp.polyu.edu.hk/ biometrics/

14. Daugman, J., Williams, G.: A Proposed Standard for Biometric Decidability. In: Proc. CardTech/SecureTech Conference, Atlanta, GA, pp. 223-234 (1996) 